\title{
Ergonomia odontológica: integrando teoria e prática para o avanço do ensino
}

Tânia Adas Saliba Rovida*; Artênio José Ísper Garbin **; Luis Fernando Dahmer Peruchini***; Ana Carolina Bernardes Machado****; Suzely Adas Saliba Moimaz*****

* Professora Doutora, Programa de Pós-Graduação em Odontologia Preventiva e Social da Faculdade de Odontologia de Araçatuba

** Professor Adjunto, Programa de Pós-Graduação em Odontologia Preventiva e Social da Faculdade de Odontologia de Araçatuba

*** Mestre em Odontologia Preventiva e Social pela Faculdade de Odontologia de Araçatuba

Mestranda em Odontologia Preventiva e Social da Faculdade de Odontologia de Araçatuba

Professora Titular, Coordenadora do Programa de Pós-Graduação em Odontologia Preventiva e Social da Faculdade de Odontologia de Araçatuba

\section{RESUMO}

O estudo objetiva descrever e analisar a experiência de uma estratégia inovadora na disciplina de ergonomia odontológica, com uso de tecnologias digitais e método ativo de ensino-aprendizagem. Foi realizado um estudo observacional, descritivo em Laboratório de Ensaios Ergonômicos de um curso de Odontologia, sobre uma estratégia de aprendizado baseado em problemas (PBL) e emprego de filmagem de atendimento clínico. Participaram 20 alunos do último ano do curso de graduação e o estudo foi desenvolvido em duas etapas: na primeira, os acadêmicos tiveram aulas expositivas dialogadas sobre princípios ergonômicos na prática odontológica para introdução do assunto; na segunda etapa, os alunos foram divididos em dois grupos: um de observadores e outro de operadores para o atendimento a pacientes. Enquanto o grupo de operadores era filmado, o outro fazia observações em tempo real, em sala anexa, com visualização direta do ambiente de atendimento através de uma janela de vidro espelhado. O entendimento dos alunos e suas respostas aos estímulos para resolução de problemas ergonômicos foram verificados, permitindo ponderar sobre as vantagens do método de ensino. Observou-se boa participação dos acadêmicos nas atividades, com apontamento dos erros, resolução dos problemas posturais, de organização de bandeja e de todo o processo de trabalho. Os alunos corrigiam sua postura durante os atendimentos realizados após as primeiras observações, mantendo-as mais adequadas durante o trabalho. Em conclusão, a metodologia empregada, aliando a aprendizagem baseada em problemas e o uso de tecnologias digitais foi importante no ensino da ergonomia odontológica.

Descritores: Educação em Odontologia. Engenharia Humana. Odontologia. Aprendizagem Baseada em Problemas. 


\section{INTRODUÇÃO}

O clássico modelo de educação bancária, advindo dos anos de 1920, coloca o professor como o único detentor de conhecimento e principal fonte de ensino, e os alunos sujeitos passivos que atuam a maior parte do tempo como ouvintes ${ }^{1}$. Com a evolução tecnológica houve enorme ganho na rapidez com que a informação é gerada e a busca pelo conhecimento deve ser uma constante e de forma diferente da que foi no passado devendo ser desenvolvida pelos acadêmicos de Odontologia, futuros profissionais da saúde, e que dificilmente seria conquistada efetivamente com as metodologias de ensino tradicionais baseadas em uma maneira geral na aquisição de conhecimentos de forma mecânica, com sua metodologia alicerçada em aulas expositivas² ${ }^{2}$. Há a necessidade de aguçar a vontade do acadêmico para raciocinar, argumentar e aprender, para que no futuro ele possa ser um profissional crítico e comprometido ${ }^{3,4}$.

As metodologias ativas de ensinoaprendizagem, desenvolvidas no Canadá ao final da década de $60,{ }^{5}$ quando inseridas durante a graduação de cursos da área da saúde, estimulam o aprendizado integrado e dinâmico. Os assuntos são estabelecidos a partir de diretrizes educacionais préestabelecidas e os problemas discutidos pelos acadêmicos após breve sessão tutorial e pesquisa prévia. O papel do professor é o de facilitador, portanto, este não ministra aulas teóricas, mas estimula a aprendizagem dos acadêmicos guiando-os a selecionar soluções pertinentes para cada situação-problema ${ }^{6,7}$. Dentre as metodologias ativas, destaca-se a Aprendizagem Baseada em Problemas (PBL, Problem-Based Learnig), que se caracteriza por trabalhar com pequenos grupos de 10 a 12 estudantes e um professor-tutor. Existe, também, a Aprendizagem Baseada em Equipes (TBL - Team-Based Learnig), que se caracteriza por grupos pequenos, com 5 a 8 estudantes ${ }^{8}$. As características básicas dos métodos ativos de ensino-aprendizagem demonstram de maneira clara a migração do "ensinar" para o "aprender", mudando o foco do professor para o acadêmico, assumindo este a responsabilidade pelo seu aprendizado, desenvolvendo, assim, a autonomia individual e valorizando o aprender a aprender ${ }^{9-11}$.

Nos anos 1980 ocorreu a introdução do ensino da ergonomia em cursos de Odontologia, como senso comum entre professores americanos; sendo Hollis ${ }^{12}$ a pioneira na inclusão da disciplina no currículo $^{13}$. A ergonomia coloca o ser humano como foco, sendo assim, avalia suas aptidões e deficiências. O ensino da ergonomia odontológica deve ser calcado nas atividades práticas do cirurgião-dentista e na solidez das normas e diretrizes ergonômicas ${ }^{14}$. Os conteúdos de ergonomia na estrutura curricular buscam identificar e promover a organização para a prática saudável da profissão, racionalizando o tempo de trabalho e contribuindo para a preservação da biossegurança; abordando conteúdos de adequação postural durante $\mathrm{o}$ atendimento clínico $^{15}$, visto que há maior prevalência de dores osteomusculares quando, durante o atendimento clínico, o cirurgião-dentista adota posturas de trabalho incorretas ${ }^{16}$. É de grande importância que o aluno se familiarize com padrões ergonômicos durante o curso, visto que é alto o índice de doenças ocupacionais durante a graduaçãa ${ }^{17}$.

$\mathrm{O}$ ensino nas escolas de Odontologia tem passado por mudanças ${ }^{18,19}$ objetivando transpor o modelo tradicional, centrado em diagnosticar, para um modelo que vise à promoção de saúde e prevenção de doenças, 
propiciando a formação de profissionais críticos e reflexivos ${ }^{6,20,21}$.

Diante do exposto, a disciplina de Ergonomia Odontológica da Faculdade de Odontologia de Araçatuba (FOA/UNESP) buscou avaliar uma estratégia de ensinoaprendizagem baseada em cenários reais desenvolvida no laboratório de ensinopesquisa.

\section{MÉTODOS}

Trata-se de um estudo descritivo, observacional, desenvolvido no laboratório de Ensaios Ergonômicos da FOA/UNESP. Este laboratório, criado para a realização de pesquisa-ensino, é dividido em dois ambientes: um consultório odontológico completo, com sistema de monitoramento por vídeo e uma sala de observação e captura das imagens com parede envidraçada, possibilitando a visualização direta do ambiente de atendimento.

A sala de atendimento é dividida em áreas segundo a International Standards Organization e a Federation Dentaire Internacionale (ISO/FDI), ${ }^{22}$ apresentando áreas demarcadas, imitando o mostrador de um relógio, conforme a figura 1 .

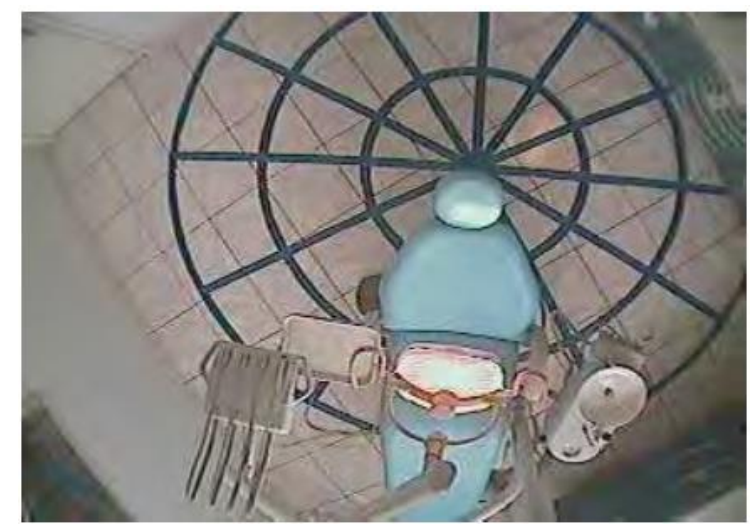

Figura 1 - Visão superior (primeira câmera) do consultório odontológico no Laboratório de Ensaios Ergonômicos da FOA/UNESP
O centro corresponde ao eixo dos ponteiros referenciando a boca do paciente deitado. Círculos concêntricos de raios de 0,5; 1,0 e 1,5 metros foram demarcados a partir do centro. Para delimitar as posições, foi convencionada que a posição de 12 horas é a indicada pela cabeça do paciente; a sala, desta forma, foi dividida em duas, onde o eixo 126h, à esquerda, é do auxiliar e de 6-12h, à direita, é a do cirurgião-dentista (figura 2).

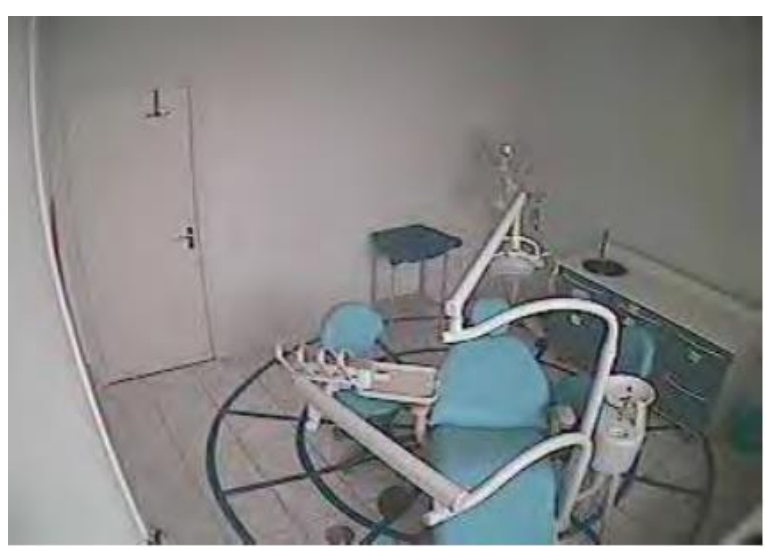

Figura 2 - Visão da terceira câmera do consultório odontológico no Laboratório de Ensaios Ergonômicos da FOA/UNESP.

O consultório possui sistema de monitoramento composto por quatro câmeras profissionais Cco Color Keep HDL (HDL, Itu, São Paulo, Brasil) com lente íris fixa, sendo uma posicionada no teto da sala de atendimento, acima do ponto central dos círculos desenhados no chão, duas nos cantos superiores da sala e uma localizada atrás do operador, quando esse se encontra em posição de 9 horas (figuras 1 e 2). Nesse estudo, as imagens captadas foram transferidas para uma central na sala de observação.

Os sujeitos da pesquisa foram 20 acadêmicos concluintes do curso de graduação em Odontologia da FOA/UNESP. Os acadêmicos foram selecionados por amostragem sistemática, sendo excluídos da 
amostra aqueles que apresentavam sintomas de distúrbios musculoesqueléticos ou que relatavam algum tipo de dor.

A participação dos indivíduos se deu de forma espontânea, após serem esclarecidos sobre os objetivos da pesquisa e que as imagens coletadas poderiam ser utilizadas em aulas e publicações científicas, aceitando participar da mesma.

O desenvolvimento do estudo seguiu as diretrizes e normas para a pesquisa envolvendo seres humanos de acordo com a Resolução CNS 466/2012.

A metodologia foi desenvolvida em duas etapas: na primeira os acadêmicos tiveram aulas expositivas e dialogadas sobre ergonomia odontológica e doenças ocupacionais, conforme previsto na estrutura curricular da instituição.

$\mathrm{Na}$ segunda etapa os estudantes foram divididos em dois grupos, um formado por observadores e o outro para o atendimento a pacientes. Enquanto esse último grupo era filmado, os demais alunos faziam as observações em tempo real, em sala anexa, com visualização direta do ambiente de atendimento.

Os procedimentos realizados foram raspagem, alisamento e polimento radicular; restaurações em resina composta; exodontias simples; biopulpectomias e necropulpectomias de dentes unirradiculares.

Os observadores eram instigados pelo professor a identificar os aspectos incorretos durante $o$ atendimento, considerando a organização do consultório e aspectos relacionados ao desenvolvimento de doenças ocupacionais.

Avaliou-se a capacidade de observação e detecção de erros posturais durante o atendimento, em tempo real, quanto ao uso inadequado dos equipamentos e instrumentos odontológicos.

Nesta etapa, as filmagens realizadas durante os procedimentos clínicos foram também projetadas em sala de aula. A partir da visualização do atendimento, os alunos identificavam os erros e os grupos reuniam-se para discutir os casos e propor soluções.

As estratégias de ensino-aprendizagem contemplaram também as seguintes ações: discussão em duplas, busca na literatura por artigos científicos sobre os assuntos levantados e discussão em grupo maior, com os demais observadores e operadores, com ativação pelo docente.

Após as discussões o docente realizava o desfecho da questão e determinava a nova dupla de operadores para a próxima sessão.

\section{RESULTADOS}

Vários erros posturais, como a inclinação das costas, inclinação e rotação da cabeça e a elevação dos membros superiores foram identificados pelo tutor durante os tratamentos endodônticos. Os alunos foram capazes de identificar estes problemas posturais e apontavam como solução o melhor ajuste do equipamento odontológico em relação ao profissional e o uso de novas tecnologias, como as lupas.

$\mathrm{Na}$ análise dos procedimentos de exodontia, o tutor identificou que o operador, na maioria das vezes, trabalhava com o tronco distante do encosto da cadeira, angulação fechada da coxa em relação à panturrilha, inclinação da cabeça e novamente com os membros superiores elevados. Grande parte dos estudantes que observavam o atendimento não conseguiu identificar a angulação das pernas como um problema. Assim, este aspecto foi levantado para discussão em grupo e definido como atividade de dispersão para estudo dirigido, visando o aprofundamento da 
temática. Na discussão final dos casos, todos os acadêmicos já identificavam este como um problema ergonômico, apontando as dores musculares, má circulação sanguínea nos membros inferiores e dores nas costas como consequências desta postura incorreta.

Durante os procedimentos restauradores as seguintes situações foram observadas: inclinação incorreta e rotação do pescoço, posição e distância inadequados do pedal em relação ao pé e mal posicionamento dos membros superiores. A posição incorreta do pé em relação ao pedal não foi identificada pelos acadêmicos, sendo esta questão instigada pelo tutor. Após as discussões, na etapa final, notou-se que os observadores já atentavam para essa situação.

Os principais problemas apresentados pelos acadêmicos em relação ao operador foram a elevação dos membros superiores, inclinação e rotação da cabeça e pescoço, inclinação das costas, angulação fechada da coxa em relação à panturrilha, inclinação da cabeça, postura inadequada da coluna e posição incorreta do pé em relação ao pedal. Com relação à disposição dos equipamentos $\mathrm{e}$ instrumentos odontológicos os erros foram desorganização da bandeja do equipo, distância excessiva da bandeja em relação ao operador e auxiliar e incorreto posicionamento do refletor (figura 3 ).

A bandeja deve ficar na posição 3/, localizada sobre o braço esquerdo da cadeira, e a unidade auxiliar localizada atrás da cadeira odontológica, equidistante do operador e auxiliar, para que ambos tenham acesso aos instrumentais e materiais sem esforços adicionais.

A grande vantagem do método empregado foi a identificação de problemas pelos próprios alunos, com a participação ativa dos mesmos nas discussões sobre a organização do processo de trabalho na prática clínica odontológica.

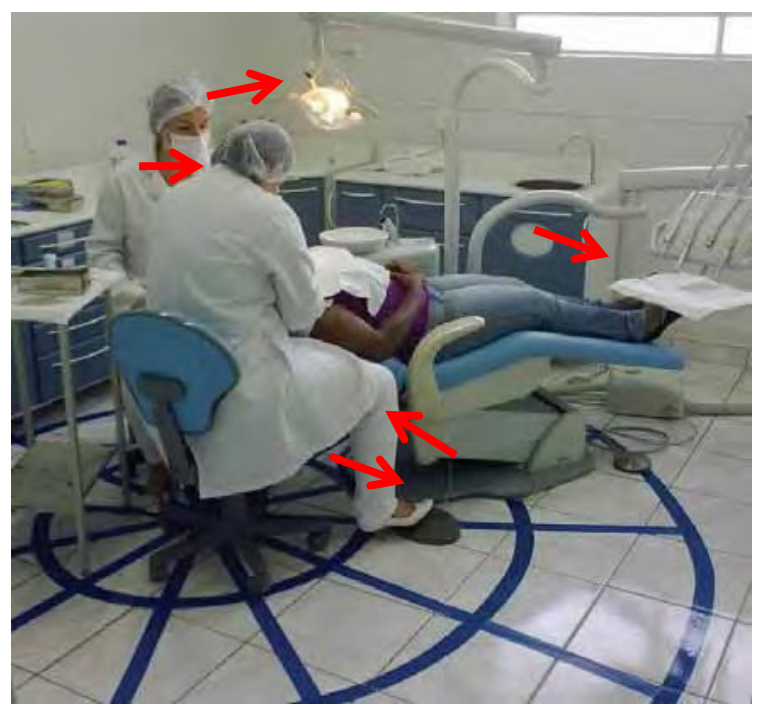

Figura 3 - Alguns dos erros mais observados: incorreto posicionamento do refletor, distância excessiva da bandeja, inclinação da cabeça, angulação fechada da coxa em relação à panturrilha e posição incorreta do pé em relação ao pedal.

O estímulo do professor fez com que os alunos buscassem as respostas em livros e principalmente em artigos científicos para aprofundamento da temática e melhor apreensão do conhecimento.

A avaliação qualitativa dos atendimentos clínicos revelou um grande avanço na percepção do desvio das normas ergonômicas e, consequentemente, na autocorreção postural.

A inclinação do tronco e a elevação dos braços destacaram-se entre as posturas incorretas mais percebidas pelos alunos durante a segunda sessão de atendimento. A observação dos acadêmicos durante a organização do material de trabalho, após a primeira experiência, também melhorou com a adequação da disposição dos materiais e instrumentais nas bandejas e a adaptação da distância destes em relação ao operador. 
Ao final do semestre foi realizada, em sala de aula, a etapa final da dinâmica de ensino para avaliação do aprendizado e percepção dos alunos sobre a estratégia empregada.

Alguns dos atendimentos gravados foram selecionados pelo professor-tutor, que estabeleceu novos problemas e os projetou para análise de todo o grupo. As consequências de cada problema identificado foram apontadas pelos próprios acadêmicos que, incentivados pelas discussões das aulas laboratoriais, aplicaram na prática os conhecimentos adquiridos.

\section{DISCUSSÃO}

Nesse estudo sobre o emprego de uma metodologia ativa de ensino-aprendizagem aplicada à ergonomia odontológica foi possível analisar o avanço gradual do conhecimento dos acadêmicos e aplicação na prática clínica.

Os cursos da área da saúde evidenciam a necessidade de mudança na maneira como se formam os profissionais, sendo estimuladas a transformarem-se na direção do ensino voltado às necessidades das demandas sociais, assim como na eficiência e relevância do seu trabalho ${ }^{23}$.

Devido à falta de experiências, quanto mais jovem é o acadêmico ou o profissional maior é o risco de desenvolver doenças ocupacionais. Os mais velhos encontrariam adaptações em seu trabalho, que acabam prevenindo $\mathrm{o}$ aparecimento de dores $\mathrm{e}$ distúrbios musculoesqueléticos ${ }^{24}$. Esta afirmação aponta a urgência do ensino da ergonomia odontológica.

Em relação à avaliação do conhecimento de acadêmicos sobre princípios ergonômicos, e se estes os colocavam em prática durante os atendimentos clínicos, constatou-se que os alunos possuíam um bom conhecimento sobre ergonomia, porém, não os utilizavam durante o trabalho.

À vista disso, considera-se ser de grande importância o incremento no ensino e na forma de avaliação, unindo a teoria à prática, pois existe uma lacuna entre as duas vertentes de ensino ${ }^{15}$.

A avaliação da ergonomia por meio de filmagem dos procedimentos permite a comparação entre os procedimentos executados, possibilitando ajustes e adequações rápidas e eficientes, estimulando a prática profissional saudável ${ }^{15}$.

Este processo pode ser aplicado também com acadêmicos durante o processo de aprendizado, como foi observado no presente estudo ${ }^{25}$.

Existem quatro tipos de ergonomia: a de concepção, que aborda a criação dos instrumentos de trabalho; a de correção, aplicada a situações reais para solução de problemas no processo de trabalho; a de conscientização, que busca capacitar o profissional a trabalhar baseado em seus princípios; e a de participação, que envolve o trabalhador buscando resolver problemas observados durante a atividade laboral.

Neste estudo empregou-se uma metodologia ativa de ensino-aprendizagem baseada nas duas últimas vertentes dessa classificação da ergonomia, enfatizando os princípios ergonômicos e trazendo $\mathrm{o}$ acadêmico para observar seu trabalho, corrigindo e otimizando sua atividade ${ }^{26}$.

A mudança na prática pedagógica traz desafios ao profissional e à instituição uma vez que se faz necessário abandonar modelos tradicionais tanto no cotidiano do professor quanto na estrutura do curso ${ }^{23}$. 


\section{CONCLUSÃO}

A estratégia ativa de ensinoaprendizagem, utilizando a filmagem de atendimentos a pacientes, em laboratório de ergonomia, possibilitou a identificação de várias situações problema, melhorando o senso crítico do acadêmico em relação à organização do trabalho na prática clínica odontológica e a sua percepção sobre a importância da ergonomia em seu cotidiano.

\section{AGRADECIMENTOS}

Agradecemos ao Conselho Nacional de Desenvolvimento Científico e Tecnológico pela disponibilização da bolsa de mestrado.

\section{ABSTRACT \\ Dental ergonomics: integrating theory and practice for teaching improvement}

This study aimed to describe and analyze an innovative strategy in the discipline of dental ergonomics, using digital technologies and active method of teaching-learning. An observational, descriptive study was performed in an Ergonomic Testing Laboratory, using the Problem Based Learning (PBL) strategy. The clinical care sessions were registered in film. Twenty undergraduate students participated and the study was developed in two stages: first, the students had a lecture on ergonomic principles in dental practice for introduction of the subject; in the second stage, they were divided into two groups, one of observers and the second of operators to patient care. While the latter group was filmed, the other made observations in real time, in an adjacent room, with direct visualization of the care environment through a mirrored glass window. There was good participation in academic activities, pointing errors, and resolution of postural problems, tray organization and the entire work process. Students corrected his stance during the care provided after the first observations, keeping the most suitable for the job. In conclusion, the strategy combining the problem-based learning and the use of digital technologies was important in the dental ergonomic education.

Descriptors: Dental Education. Human Engineering. Dentistry. Problem-Based Learning.

\section{REFERÊNCIAS}

1. Arruda MP, Portal LLF. Saberes e fazeres docentes: o dilema da reforma do pensamento e da prática pedagógica do educador do século XXI. Percursos. 2012;13(1):199-210.

2. Manogue M, McLoughlin J, Christersson C, Delap E, Lindh C, Schoonheim-Klein $\mathrm{M}$, et al. A curriculum structure content, learning and assessment in European undergraduate dental education. Eur $\mathbf{J}$ Dent Educ. 2001;15;133-41.

3. Freitas VP, de Carvalho, RB, Gomes MJ, Figueiredo MC, Faustino-Silva DD. Mudança no processo ensino aprendizagem nos cursos de graduação em odontologia com utilização de metodologias ativas de ensino e aprendizagem. RFO. 2009;14(2):163-7.

4. Aquilante AG, Tomita NE. O estudante de Odontologia e a educação. Rev ABENO. 2005;5(1):6-11.

5. Neville AJ. The problem-based learning tutor: Teacher? Facilitator? Evaluator? Med Teach. 1999; 21(4):393-401.

6. Xavier LN, de Oliveira GL, Gomes AA, Machado MFAS, Eloia SMC. Analisando as metodologias ativas na formação dos profissionais de saúde: uma revisão integrativa. SANARE. 2014;13(1):76-83.

7. Lombardo I. Reflexões sobre o planejamento do ensino de Odontologia. Rev ABENO. 2001; 1(1):17-24.

8. Farias PAM, Martin ALAR, Cristo CS. Aprendizagem ativa na educação em saúde: percurso histórico e aplicações. Rev Bras Educ Med. 2015;39(1):143- 
58.

9. Troncon LEA, Bollela VR, Borges MC, Rodrigues MLV. A formação e o desenvolvimento docente para os cursos das profissões da saúde: muito mais que o domínio de conteúdos. Medicina. 2014;47(3):245-8.

10. Noro LRA, Farias-Santos BCS, Settede-Souza PH, Cruz RKS, Pinheiro IAG, Borges REA, et al. O professor (ainda) no centro do processo ensinoaprendizagem em Odontologia. Rev ABENO. 2015;15(1):2-11.

11. Saliba NA, Moimaz SAS, Chiaratto RA, Tiano AVP. A utilização da metodologia PBL em Odontologia: descortinando novas possibilidades ao processo ensino-aprendizagem. Rev Odonto Ciênc. 2008;23(4):392-6.

12. Hollis M. Safer lifting for patient care. 2ed. Oxford: Blackwell Scientific Publications; 1985.

13. Troup JDG, Rouhala HH. Ergonomics and training. Int $\mathbf{J}$ Nurs Stud. 1987;24(4):325-30.

14. Garbin AJI, Garbin CAS, Diniz DG. Normas e diretrizes ergonômicas em odontologia: o caminho para a adoção de uma postura de trabalho saudável. Rev Odontol Univ Cid São Paulo. 2009;21(2):155-61.

15. Garbin AJI, Garbin CAS, Ferreira NF, Saliba MTA. Ergonomics and the dentist: an evaluation of clinical practice by filming analysis. Rev Odonto Ciênc. 2008;23(2):130-3.

16. Garbin AJI, Garbin CAS, Arcieri RM, Rovida TAS, Freire ACGF. Musculoskeletal pain and ergonomic aspects of dentistry. Rev Dor. 2105;16(2):90-5.

17. Vieira AJO, Julião Filho CAB, Firmino RT, Garcia AFG, Menezes VA. Conhecimento de ergonomia e desordens osteomusculares entre estudantes de Odontologia. RFO. 2014;19(3):304-10.

18. Reis SMAS, Cicillini GA. Práticas docente no ensino odontológico: aproximações e distanciamentos das diretrizes curriculares nacionais. Rev Ib-Am Estudos Educ. 2011;6(2). Disponível em: http://seer.fclar.unesp. br/iberoamericana/article/view/4878

19. Cunha FS, Silva AE, Larentis NL, Fontanella VRC, Nevado RA. Proposta de uma nova abordagem pedagógica para a Disciplina de Informática aplicada à Odontologia. Rev ABENO. 2005;5(2):102-8.

20. Morita MC, Kriger L. Mudanças nos cursos de Odontologia e a interação com o SUS. Rev ABENO. 2004;4(1):17-21.

21. Rodrigues CRC, Silva MAM. O impacto dos cenários de prática propostos pelo Pró-Saúde na formação em odontologia. Rev ABENO. 2012;12(2):219-26.

22. International Standards Organization ISO. ISO/TC 106/SC 6 N 411: 2006.

23. Cyrino EG, Toralles-Pereira ML. Discovery-based teaching and learning strategies in health: problematization and problem-based learning. Cad Saúde Públ. 2004;20(3):780-8.

24. Leggat PA, Kedjarune U, Smith DR. Occupational health problems in modern dentistry: a review. Ind Health. 2007;45(5):611-21.

25. Gottlieb R, Lanning SK, Gunsoley JC, Buchanan JA. Faculty impressions of dental students' performance with and without virtual reality simulation. $\mathrm{J}$ Dent Educ. 2011;75(11):1443-51.

26. Wisner A. La méthodologie em ergonomie: d'hier, à aujourd'hui. Perform Hum Tech. 1990;50:32-8.

Correspondência para:

Ana Carolina Bernardes Machado

e-mail: anab.odonto@gmail.com

Rua José Bonifácio, 1193 - Seção de Pós-

Graduação

16015-050, Vila Mendonça, Araçatuba - SP 